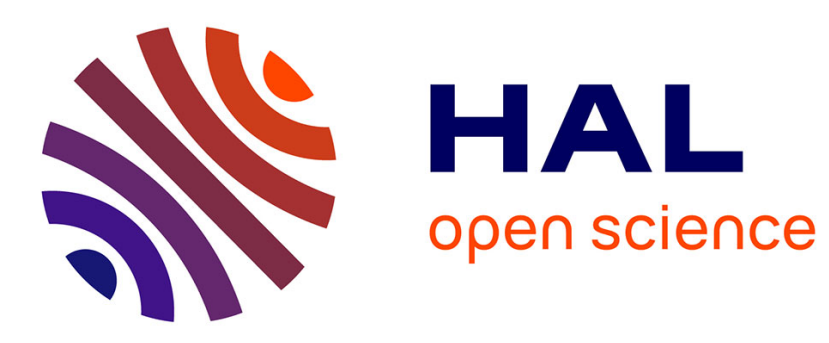

\title{
Improving the drying of Propionibacterium freudenreichii starter cultures
}

Romain Jeantet, Gwénaël Jan

\section{To cite this version:}

Romain Jeantet, Gwénaël Jan. Improving the drying of Propionibacterium freudenreichii starter cultures. Applied Microbiology and Biotechnology, 2021, 105, pp.3485-3494. 10.1007/s00253-02111273-3 . hal-03258328

\section{HAL Id: hal-03258328 \\ https://hal.inrae.fr/hal-03258328}

Submitted on 11 Jun 2021

HAL is a multi-disciplinary open access archive for the deposit and dissemination of scientific research documents, whether they are published or not. The documents may come from teaching and research institutions in France or abroad, or from public or private research centers.
L'archive ouverte pluridisciplinaire HAL, est destinée au dépôt et à la diffusion de documents scientifiques de niveau recherche, publiés ou non, émanant des établissements d'enseignement et de recherche français ou étrangers, des laboratoires publics ou privés.

\section{(c)(1)}

Distributed under a Creative Commons Attribution| 4.0 International License 


\title{
Improving the drying of Propionibacterium freudenreichii starter cultures
}

\author{
Romain Jeantet ${ }^{1} \cdot$ Gwénaël $\operatorname{Jan}^{1}$ (I) \\ Received: 28 December 2020 / Revised: 30 March 2021 / Accepted: 5 April 2021 / Published online: 22 April 2021 \\ (C) The Author(s), under exclusive licence to Springer-Verlag GmbH Germany, part of Springer Nature 2021
}

\begin{abstract}
Propionibacterium freudenreichii is a beneficial food-grade actinobacterium, widely implemented, and thus consumed, in various food products. As the main application, P. freudenreichii is used as a cheese-ripening starter, mostly in hard type cheeses. Indeed, during manufacture of "Swiss-type" cheeses (or opened-body cheeses), the technological process favors propionibacteria growth, as well as the corresponding propionic fermentation. This leads to the characteristic flavor of these cheeses, through the release of short chain fatty acids and through lipolysis, as well as to their specific texture. To fulfil this ripening, massive amounts of propionibacteria are industrially produced, dried and stored, prior to cheese making. Furthermore, $P$. freudenreichii is commercialized in various probiotic food supplements aiming at preserving intestinal health and comfort, in line with its ability to produce beneficial metabolites (short chain fatty acids, vitamins), as well as immunomodulatory compounds. Other industrial applications of $P$. freudenreichii include the production of food-grade vitamins of the B group, of trehalose, of conjugated linoleic acid, and of biopreservatives. For these different applications, maintaining survival and activity of propionibacteria during production, drying, storage and finally implementation, is crucial. More widely, maintaining live and active probiotic bacteria represents a challenge as the market for probiotic products increases. Probiotic bacteria are, for a bulk majority, freeze-dried, but spray drying is also more and more considered. Indeed, this process is both continuous and more cost-efficient, as it utilizes less energy compared to freeze-drying; on the other hand, it exposes bacteria to higher heat and oxidative stresses. Apart from process optimization and strain selection, it is possible to enhance the resistance of bacteria by taking advantage of their adaptation capacity. Indeed, $P$. freudenreichii stress tolerance can be boosted by different pretreatments applied before the drying step, thus considerably increasing its final survival. In particular, adaptation to hyperosmotic conditions improves stress tolerance, while the presence of osmoprotectants may mitigate this improvement. Thermal adaptation also modulates tolerance towards these technological challenges. The composition of the growth medium, including the ratio between the carbohydrates provided and the non-protein nitrogen, plays a key role in driving the accumulation of osmoprotectants. This, in turn, determines $P$. freudenreichii tolerance towards different stresses, and overall towards both freeze-drying and spray-drying. As an example, the accumulation of trehalose enhances its spray-drying survival, while the accumulation of glycine betaine enhances its freeze-drying survival. Growth of propionibacteria in hyperconcentrated whey was used to trigger multiple stress tolerance acquisition, underpinned by overexpression of key stress protein, accumulation of cytoplasmic storage compounds, and leading to enhanced spray-drying survival. A simplified process, from cultivation to atomization, was developed by using whey as a 2 -in-1 medium in which propionibacteria were grown, protected and dried with minimal cell death. This innovative process was then subjected to scaling up at the industrial level. In this aim, a gentle multi-stage drying process offering mild drying conditions by coupling spray drying with belt drying, led to final probiotic survival close to $100 \%$ when stress tolerance acquisition was previously implemented. Such innovation opens new avenues for the efficient, cost-effective and sustainable development of new probiotic production technologies, as well as probiotic application in the context of food and feed.
\end{abstract}

\section{Key points}

- Propionibacteria acquire multi-stress tolerance when grown in hyper-concentrated whey.

- Spray drying of osmo-adapted probiotic bacteria is possible with limited cell death.

Gwénaël Jan

gwenael.jan@inrae.fr

1 STLO, INRAE, Institut Agro, 35042 Rennes, France
- A two-in-one drying method is developed to grow and dry probiotic bacteria in the same matrix.

Keywords Probiotic - Spray drying - Stress - Adaptation · Propionibacteria $\cdot$ Starter 


\section{Introduction}

Propionibacteria are beneficial bacteria with various biotechnological applications. They are Gram-positive, non-motile, aerotolerant, with a high GC content. They are mainly isolated from raw milk, cheese and other fermented products, including silage. They are used as ripening cultures for the manufacture of Swiss-type cheeses, as probiotics and as vitamin producers in the food industry. Their energetic metabolism relies on propionic fermentation, leading to the release of propionic acid as a major end product by using unusual transcarboxylase enzymes. Their propionic fermentation leads to the characteristic sweet and nutty flavour, and to the formation of holes (so-called eyes) in such cheeses. They were first described by Sigurd OrlaJensen and Eduard von Freudenreich at the beginning of the twentieth century (von Freudenreich and Orla-Jensen 1906), while propionic fermentation had been studied by Louis Pasteur and Albert Fitz (Fitz 1878; Pasteur and Faulkner 1879). They are described as pleomorphic rods, or small cocci, arranged in pairs, short chains or clusters resembling Chinese characters (Thierry et al. 2011). They belong to the actinomycetales order. This order comprises bacteria described with a mycelium-like aspect, colonizing various types of environments, including animal hosts and environmental niches such as soil, and known for their prolific production of small molecules, including antimicrobials. Dairy propionibacteria, including the most used species Propionibacterium freudenreichii, belong to the family of Propionibacteriaceae, which is subdivised into three genera: Propionibacterium spp, Acidipropionibacterium spp and Cutibacterium spp (Turgay et al. 2020). In the previous bacterial classification, the genus Propionibacterium used to include cutaneous species such as $P$. acidifaciens, $P$. acnes, $P$. australiense, $P$. avidum, $P$. granulosum, $P$. propionicum and $P$. humerusii. Other dairy (or classical) species of this Propionibacterium genus were $P$. acidipropionici, $P$. cyclohexanicum, $P$. thoenii, $P$. jensenii, $P$. microaerophilum and $P$. freudenreichii. It should be noted that the cutaneous species may constitute opportunistic pathogens, while dairy species are harmless. This classification was however modified, so that the Propionibacterium genus today comprises the $P$. freudenreichii and $P$. cyclohexanicum harmless species. The cutaneous species now belong to the Cutibacterium genus, clearly distinct from dairy propionibacteria. This minireview will focus on Propionibacterium freudenreichii, the main beneficial species used for human consumption. It should be noted, however, that Acidipropionibacterium jensenii, A. acidipropionici and $A$. thoenii may also occur in raw milk and in cheeses such as "Swiss-type" cheeses (or opened-body cheeses). The long-documented history of safe use of $P$. freudenreichii and A. acidipropionici conferred to these two species the generally recognized as safe (GRAS) status in the USA, as well as the qualified presumption of safety (QPS) one in the EU. Furthermore, $P$. freudenreichii is more and more considered for its probiotic use in humans (Rabah et al. 2017) and gained recent attention as it was recently described as a human commensal bacterium, acting as a symbiont responsible for immune modulation (Colliou et al. 2017). There is a global growing demand for fermented foods, for functional foods and for probiotic foods supplements. P. freudenreichii has the potential to play a role in the answer to this growing demand, in addition to its wide utilisation as a starter in the food industry.

\section{Propionibacterium freudenreichii is a useful industrial starter}

P. freudenreichii can ferment a variety of substrates including carbohydrates (such as lactose), polyols (such as glycerol and erythritol), and organic acids (such as lactate, preferred carbon and energy source). Carbohydrates and organic acids are transformed into pyruvate, a metabolic node molecule, which may be used either for the NADH-generating synthesis of acetate, or for the NADH-consuming synthesis of propionate (Fig. 1). P. freudenreichii maintains its redox balance by modulating these two pathways. Hence, fermentation of 3 lactates results in the production of 2 propionates, 1 acetate and $\mathrm{CO}_{2}$, as described in the Fitz equation (Piveteau 1999). This propionic fermentation, and thus $P$. freudenreichii, are needed to achieve the characteristic eyes, as well as the typical sweet and nutty flavour of opened-body cheeses. Propionic and acetic acids, in particular, are responsible for a sweet and slightly sour flavour. Propionibacteria furthermore convert amonoacids to flavour compounds including carboxylic acids and alcohols. The aminoacids catabolites 2-methylbutyrate, 3methylbutyrate and 2-methylpropionate add cheesy and sweaty notes to the cheese (Thierry et al. 2002; Thierry et al. 2004). Propionibacteria also release succinate which, together with glutamate and propionate, participates to the umami taste of cheese (Fröhlich-Wyder and Bachmann 2004; Turgay et al. 2020). They are also responsible for lipolysis in cheese, i.e. the release of free fatty acids from milk fat triglycerides, which in turn serve as precursors of aroma compounds (Dherbecourt et al. 2010a; Yee et al. 2014). They perform lipolysis thanks to a secreted esterase (Dherbecourt et al. 2010b). Finally, $P$. freudenreichii produces esters, typical fruity aroma compounds, from alcohols and acids, using both esterification and alcoholysis reactions (Turgay et al. 2020). It is also responsible for the formation of eyes, which requires saturation of the cheese matric with $\mathrm{CO}_{2}$ and the presence of nuclei in the cheese curd.

P. freudenreichii is naturally present in soil, in silage, in the rumen and intestine of ruminants, and thus in raw milk, but at low populations. Traditional PDO (Protected Designation of Origin) cheeses such as Comté may undergo spontaneous, yet limited, propionic fermentation. However, it is added at initial doses of $10^{3}$ to $10^{4}$ CFU. $\mathrm{mL}^{-1}$ to milk in the manufacture of 


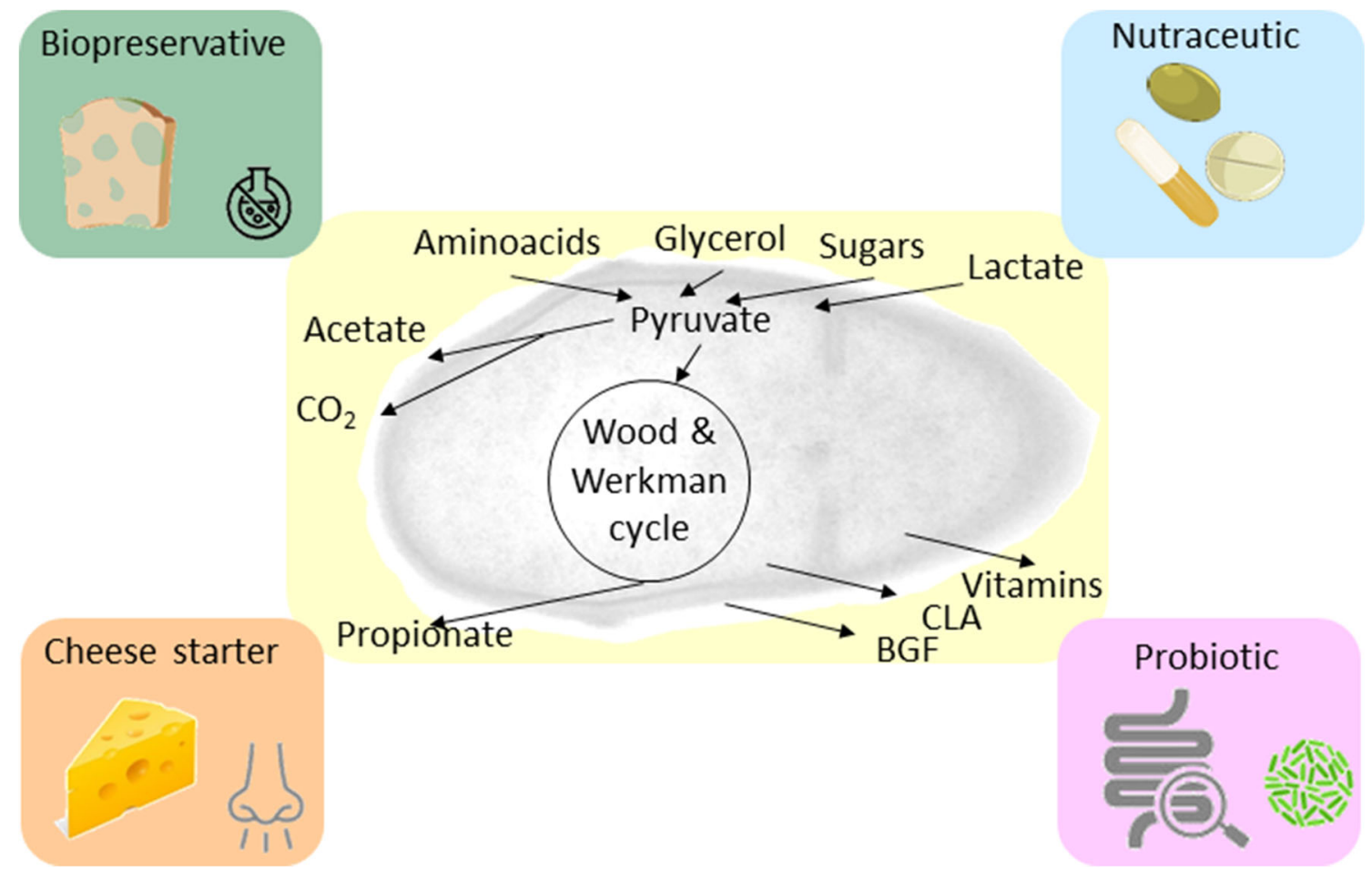

Fig. 1 Propionibacteria are multipurpose beneficial bacteria. Their specific fermentative metabolism relies on the Wood and Werkman cycle. They ferment various substrates including lactate, sugars, glycerol and aminoacids. Whatever the fermented carbon source, they produce beneficial metabolites. The short chain fatty acids acetate and propionate are responsible for cheese flavor and for the antimicrobial

Emmental, to reach a final population of $10^{8}$ to $10^{9} \mathrm{CFU}$ per gram of cheese. The bulk majority of starters comprising $P$. freudenreichii is nowadays produced under the form of dried powders. Indeed, before the development of concentrated and dry starter cultures, commercial starter cultures were originally provided under a liquid form. Industrials had to subculture in order to make intermediate and bulk starters for inoculation of fermentations. Further development in biomass production provided frozen and freeze-dried cultures for "direct-to-vat" inoculation. Dried starter cultures eliminate inplant subculturing, reduce costs associated with bulk culture preparation, reduce the risk of bacteriophages infection, as well as microbial contamination.

\section{Propionibacterium freudenreichii is a beneficial probiotic bacterium}

As aforementioned, the main species, $P$. freudenreichii, is consumed in large amounts in some cheeses and is largely recognized as safe. It is moreover considered as a beneficial commensal of the healthy human gut microbiota (Colliou et al. 2017; Ge et al. 2019) and as a probiotic (Rabah et al. 2017). Probiotic microorganisms are defined as live microorganisms, which, when administered in adequate amounts, properties used in food-grade biopreservatives. Vitamins of the B group, bifidogenic growth factor (BGF) and conjugated linoleic acid (CLA) contribute to their use as nutraceutical producers. Reported modulation of the gut physiology, microbiota and immune response contribute to their potential as probiotics

confer a health benefit to the host (FAO/WHO 2002). Probiotic microorganisms, owing to their ability to modulate the gut microbiota and/or the gut physiology, are studied in humans for their potent role in the management of intestinal dysbiosis, antibiotic-related diarrhoea, Helicobacter pylori infections, traveler's diarrhoea, recurrence of Clostridium difficile, inflammatory bowel disease such as ulcerative colitis, and irritable bowel syndrome (Sniffen et al. 2018). The dairy propionibacterium $P$. freudenreichii is commercialized as a probiotic microorgansim in various countries including France (Sécuril, Yalacta), Finland (PJS, Valio) and Japan (BGF, Meiji), in food supplements aiming at preserving intestinal health and comfort. It indeed constitutes a promising probiotic candidate, although it is less studied that lactobacilli or Bifidobacteria. In humans, it was shown to survive during the transit through the digestive tract (Bouglé et al. 1999; Jan et al. 2002b; Suomalainen et al. 2008). In the human digestive tract, its presence was shown to maintain an active propionic fermentation (Hervé et al. 2007) and to modulate intraluminal short chain fatty acids in the favour of propionate (Jan et al. 2002b). Consumption of $P$. freudenreichii modulates the human gut microbiota in the favour of bifidobacteria (Seki et al. 2004; Mitsuyama et al. 2007). Modulation of the intestinal motility was also reported (Bouglé et al. 1999), as well as alleviation of constipation (Hojo et al. 2002). In human 
neonates, its presence within the gut microbiota, allowed by maternal breast-feeding, coincides with a reduced probability to develop necrotizing enterocolitis (Colliou et al. 2017). Accordingly, a Japanese human pilot study indicated alleviation of ulcerative colitis symptoms, as a result of the consumption of dried cultures of $P$. freudenreichii (Mitsuyama et al. 2007).

The probiotic potential of $P$. freudenreichii has been explored at the molecular level. It involves the active production of small metabolite molecules playing a physiological role (short chain fatty acids, vitamins), and of surface proteins responsible for adhesion and immune modulation. The short chain fatty acids, including acetate and propionate, attracted the attention of scientists in the context of beneficial effects. Indeed, enhancing their intra-intestinal concentrations is thought to help in preventing gastro-intestinal dysfunction, obesity and type 2 diabetes mellitus (Blaak et al. 2020). $P$. freudenreichii was shown to induce in vitro apoptosis, the intrinsic programmed cell death, via acetate and propionate acting on colorectal carcinoma cells' mitochondria (Jan et al. 2002a). Selected strains of $P$. freudenreichii, able to adapt digestive stresses and to produce propionate in situ (Lan et al. 2007), were further shown to favour apoptotic depletion of colon cancer cells in mutagenized rats (Lan et al. 2008). Accordingly, propionibacterial metabolites were shown to synergize the action of chemotherapeutic molecules in vitro, opening perspectives for targeted functional foods (Cousin et al. 2016).

$P$. freudenreichii was furthermore described as a nutraceutical producer (Hugenholtz et al. 2002). Indeed, it produces several nutraceutical molecules beneficial to human health, such as B vitamins, including cobalamin (B12) and folic acid (B9) (Fig. 1). It utilizes vitamin B12 as a cofactor of a key step of propionic fermentation, i.e. the conversion of succinyl-CoA into methylmalonyl-CoA. The biosynthesis of B12 starts with glutamate, involves 30 genes and proceeds through the anaerobic pathway, regardless of the aerobic and anaerobic conditions of incubation (Iida et al. 2007). It is used in the industry in order to produce food supplements, as it is the only B12 producer known to be a GRAS bacterium (Deptula et al. 2015; Rabah et al. 2017). P. freudenreichii was further shown to produce and release DHNA, 1,4-dihydroxy-2-naphtoic acid, a molecule described as a bifidogenic growth stimulator (Isawa et al. 2002). Accordingly, consumption of dried supernatants of $P$. freudenreichii ET-3 cultures, rich in DHNA, provided by the Japanese company Meiji, enhanced fecal Bifidobacteria population in human healthy volunteers (Hojo et al. 2002; Seki et al. 2004; Mitsuyama et al. 2007). DHNA, a precursor in the biosynthesis of vitamin $\mathrm{K}$ (Furuichi et al. 2006), indeed has a potential application as a "prebiotic" molecule used to enhance the intestinal population of bifidobacteria. Another promising property is the ability of P. freudenreichii to convert linoleic acid (LA) into conjugated linoleic acid (CLA). More precisely, it produces the isomer cis 9 ,trans-11 octadecadienoic, referred to as rumenic acid (McIntosh et al. 2009). Animal and clinical studies reported the anti-inflammatory effects of conjugated fatty acids, including rumenic acid, by acting as PPAR $\gamma$ agonists (Penedo et al. 2013; Yuan et al. 2015; Viladomiu et al. 2016). Dairy propionibacteria, including $P$. freudenreichii, are also known to produce high amounts of trehalose, a non-reducing disaccharide. In humans, trehalose consumption improved glucose tolerance, decreased the progression to insulin resistance, and decreased systolic blood pressure, indicating that it induces systemic benefits (Mizote et al. 2016; Le Lay et al. 2016). Finally, dairy propionibacteria produce anti-microbial molecules and are used as food grade biopreservatives. They produce antifungal acids including propionic acid as the main antimicrobial, as well as succinic, acetic and 3-phenyllactic acid acids (Lind et al. 2005; Lind et al. 2007). Propionibacterial bacteriocins, antimicrobial peptides, inhibit propionibacteria, Gram-positive and Gram-negative bacteria, as well as yeasts and molds (Rabah et al. 2017). In line with the production of these different antimicrobials, dairy propionibacteria were shown to supress the growth of undesirable bacteria and molds in many foods, prolonging their shelf-life. Skim milk fermented by $P$. freudenreichii is commercialized as Microgard ${ }^{\mathrm{TM}}$ product (Du pont Danisco) as a food-grade biopreservative (Al-Zoreky et al. 1991). In addition, fermentation of whey by $P$. freudenreichii leads to the production of Inhibit 3600 Dairy $^{\mathrm{TM}}$ (Mezzoni Foods), a natural shelf-life extender (Turgay et al. 2020).

Concerning modulation of the immune response and of inflammation by surface proteins, a pioneer study evidenced promising immunomodulatory properties in $P$. freudenreichii (Foligné et al. 2010). Selected strains induced the release of the regulatory cytokine IL-10 in blood mononuclear cells PBMCs. Consumption of these strains protect mice from chemically induced colitis, and furthermore delays colonization of the gut and colitis induced by pathogenic Citrobacter rodentium. Noteworthy, this property is highly strain-dependent and only selected strain induce this response (Foligné et al. 2013). Selective extraction of $P$. freudenreichii surface proteins abrogates this immunomodulatory property (Le Marechal et al. 2015). A comparative study of strains, combining genomics, transcriptomic and proteomics, further indicated the key role of surface proteins SlpB (Deutsch et al. 2017). Inactivation of the corresponding $\operatorname{slp} B$ gene abrogates $P$. freudenreichii adhesive and immunomodulatory properties. Finally, SlpB protein was purified and it induces expression of IL-10 in cultured colonocytes (FLR et al. 2019).

In vivo, consumption of $P$. freudenreichii-fermented products delivers metabolically active propionibacteria to the pig's gut (Cousin et al. 2012b) and triggers enhanced growth and attenuated inflammatory response of the gut epithelium to LPS, with lower levels of IL-8 (Cousin et al. 2012a). It 
modulates the gut-associated cellular immune response with enhanced population of Treg lymphocytes and reduced proinflammatory response of PBMCs to LPS (Rabah et al. 2018). In mice, consumption of such products protects from colitis, whether P. freudenreichii is consumed alone (Plé et al. 2015), or in combination with a L. delbrueckii strain (Plé et al. 2016). In particular, consumption of $P$. freudenreichii limited the increase in the colic macroscopic Wallace score, the histopathological Ameho score, the weight loss, blood inflammatory markers, colic expression of inflammatory and oxidative stress markers, as well as the overall intensity of the disease (Foligné et al. 2010; Plé et al. 2015; Plé et al. 2016; Rabah et al. 2020). These results open new perspectives for the development of fermented foods and of food supplements in order to prevent and to help in treating intestinal disorders linked with inflammation. While new fermented foods will require innovative design of functional food, food supplements are still limited by the available drying technologies, acting as a bottleneck for cheap and sustainable production of stable dried probiotic products.

\section{Adaptation of $P$. freudenreichii ameliorates its stress tolerance and drying survival}

As dairy starters and as probiotics, propionibacteria are exposed to technological and digestive stresses, and stress adaptation constitutes a bottleneck for their efficacy. Early investigations revealed that $P$. freudenreichii was constitutively susceptible and suffered massive cell death upon exposure to technological and digestive stresses. However, pre-exposition to moderate doses of stress conferred remarkable stress tolerance, a process known as stress adaptation. As an example, acid adaptation, i.e. exposure to $\mathrm{pH}$ values between 4 and 5, encountered in many fermentation processes, triggers acid tolerance response, leading to survival to $\mathrm{pH}$ values down to 2 (Jan et al. 2000; Jan et al. 2001). This relies on the induction of adaptation proteins involved in DNA synthesis and repair, in propionic fermentation, as well as ATP-dependent proteases and chaperons (Jan et al. 2001). Similarly, naive $P$. freudenreichii cells experience massive cell death when exposed to concentrations of bile salts encountered within the intestine. This mortality can be supressed, provided that $P$. freudenreichii is exposed to lower doses of bile salts, an adaptive response which relies on the induction of proteins stress sensing, signal transduction, oxidative stress remediation and detoxification (Leverrier et al. 2003). Moreover, thermal adaptation confers cross-protection towards acid, bile salts and heat challenges, by inducing general stress chaperones and proteins involved in SOS response (Leverrier et al. 2004). Osmotic adaptation, i.e. adaptation of $P$. freudenreichii to elevated osmotic pressure, was early demonstrated and involves intracellular accumulation of endogenous osmoprotectants such as glutamate and trehalose and exogenous ones including glycine betaine, dimethylsulfoniopropionate and dimethylsulfonioacetate (Boyaval et al. 1999; Cardoso et al. 2007). Later, it was shown that osmoadaptation (at elevated concentrations of salt) in the absence of exogenous osmoprotectant molecules (in a chemically defined medium) enhances $P$. freudenreichii heat, oxidative and acid stress tolerance, as well as survival upon freeze-drying. By contrast, addition of glycine betaine as exogenous osmoprotectant suppressed stress tolerance, while restoring optimal growth (Gaucher et al. 2019a). The bottleneck of the freeze-drying process is the limited survival of bacteria during drying and storage. Growing of $P$. freudenreichii under hyperosmotic constraint, triggering osmoadaptation, was further combined with acid or thermal pre-treatments. Such combinations in turn triggered intracellular accumulation of stress proteins, of compatible solute glycine betaine, as well as modulations of membrane fatty acids composition. These rearrangements led to enhanced survival of $P$. freudenreichii upon freeze-drying (Gaucher et al. 2019c). Spray drying constitutes a more productive and efficient way to produce dried cultures, but a more stressing one, imposing severe oxidative and thermal challenges. Modulation of the growth conditions, heat pre-treatment and osmotic pretreatment was optimized to enhance survival of $P$. freudenreichii under technological constraints. Carbohydrate addition, coupled to heat pre-treatment, triggered accumulation of glycine betaine and of trehalose, which in turn provided high tolerance towards spray drying and storage (Gaucher et al. 2019b). Finally, hyperosmotic growth conditions can be modulated to adjust the intracellular compatible solutes content. Indeed, modulating the ratio between carbohydrates and non-protein nitrogen, during growth, modulates the ratio between glycine betaine and trehalose, and thus $P$. freudenreichii tolerance towards freeze- and spray-drying. Trehalose accumulation correlates with enhanced spray-drying survival, and glycine betaine with enhanced freeze-drying survival (Gaucher et al. 2020). In line with these results, a new growth medium was developed for $P$. freudenreichii. It consists of hyperconcentrated sweet whey, i.e. at 20 to $30 \%$ dry matter, instead of $5 \%$. Growth in this medium led to remarkable enhanced multistress tolerance acquisition, overexpression of key stress proteins, together with intracellular accumulation of storage molecules glycogen and polyphosphates and of trehalose. This, in turn, led to enhanced survival upon spray drying (Huang et al. 2016b). These results allow the development of new drying technologies, from bench to industrial pilot scale.

\section{New drying technologies are developed}

Freeze-drying is a discontinuous and energy-consuming process that generally allows high cell survival. However, the specific energy consumption of dairy bacteria freeze-drying is more than 10 times higher than that of spray-drying (Schuck 
et al. 2013). The latter, on the other hand, is more energyefficient, but may cause massive cell death because of heat, osmotic and oxidative stress exposition. Indeed, such harsh conditions, in particular at the last stage of drying, limit the use of spray-drying for the production of probiotics (Fu and Chen 2011). Many studies thus aimed at improving this process by limiting bacterial death during spray-drying (Broeckx et al. 2016). In our experience, dairy propionibacteria including $P$. freudenreichii are freeze-dried without major cell damage, so more than $50 \%$ survival is classically observed, whatever the growth medium used (Gaucher et al. 2019c). However, the first attempts to spray-dry $P$. freudenreichii without specific adaptation after growth in YEL medium led to less than $4 \%$ survival (Huang et al. 2016b). The remarkable ability of dairy propionibacteria, including $P$. freudenreichii and A. acidipropionici, to respond to stressing environmental conditions and to acquire enhanced stress tolerance allowed to develop new drying technologies, based on spray-drying, where fine-tuned adaptation leads to reduced bacterial death during drying. In particular, the contact with atmospheric oxygen and with temperatures in the range $45^{\circ} \mathrm{C}$ to $75^{\circ} \mathrm{C}$ prevailing during the spray drying process constitute the main challenges responsible for bacterial death, thus limiting the industrial production of viable probiotics. A multistage drying process was first developed to circumvent the use of too high temperatures (Schuck et al. 2013). It allowed to dry A. acidipropionici with limited bacterial death in a semi industrial scale spray drying process, while implementing low temperatures, and limiting stickiness occurrence by the use of a crystallizer or a belt drying step. In this process, propionibacteria were cultured in 13.5 $(\mathrm{w} / \mathrm{v})$ dry mater acid whey, and then concentrated by membrane ultrafiltration. Sweet whey permeate powder was then added prior to spray drying. The spray dryer was coupled to a crystallizer followed by a vibro-fluidizer, which contributed to water elimination, so that mild spray drying parameters could be fixed upstream. Indeed, the inlet temperature was set at $130^{\circ} \mathrm{C}$, while the outlet one was $60^{\circ} \mathrm{C}$.

Later, spray drying of dairy propionibacteria was further enhanced. Hyperconcentrated sweet whey was used to culture $P$. freudenreichii, providing a two-in-one medium to grow and dry propionibacteria. Dry matter was increased to 20 and $30 \%$ $(\mathrm{w} / \mathrm{v})$. Propionibacteria grew in this medium to reach higher populations than in isotonic whey, and without the need of adding growth factors. They adapted to the high osmolarity, accumulated compatible solutes and storage compounds, and subsequently reached elevated levels of stress tolerance (see above). The limited amount of water in these cultures further reduced the amount of thermal energy needed to evaporate water. A laboratory scale minor spray dryer (evaporation capacity in the range 3-5 kg.h $\mathrm{h}^{-1}$ ) was first used to dry such cultures with up to $80 \%$ survival of propionibacteria as a proof of concept for patenting. The stability of the survival in the obtained powders, in terms of viability during storage at $4^{\circ} \mathrm{C}$, was also increased as a result of growth in hyperconcentrated sweet whey. Thus, double use of hyperconcentrated sweet whey improved the biomass production and the viability of probiotic propionibacteria (Huang et al. 2016a). This process was then scaled up using a semi industrial pilot spray dryer (evaporation capacity in the range $70-120 \mathrm{~kg} \cdot \mathrm{h}^{-1}$ ). More precisely, a multistage process coupling spray drying with belt- and fluid-bed drying and thus allowing low temperature conditions was proposed (Huang et al. 2017). In the final product, the propionibacterial viability was improved, reaching up to $100 \%\left(>10^{9} \mathrm{CFU} \mathrm{g}^{-1}\right)$. Moreover, the process induced a higher tolerance towards simulated intestinal fluids; compared to fresh $P$. freudenreichii cultures. The stability of $P$. freudenreichii viability, within the powders, was monitored during 6 months of storage. This evidenced that both storage temperature and powder moisture content/water activity determine the stability the probiotic.

Overall, these studies pave the route for optimizing the survival of bacteria among spray drying. As summarized in Fig. 2, the growth on concentrated culture medium should be recommended as in a generic way it rises the stress resistance of bacteria through osmoadaptation mechanisms (1). The grown and stress adapted bacteria can be separated in a conventional way, and the bacterial paste can be rinsed and resuspended in a drying medium compatible with the market applications (2)). However, it is still possible to directly spray dry the culture if the culture medium is food grade and market compatible, which makes the process simpler and the contamination risk lower. Last, multistage drying should be recommended to lower inlet/outlet temperature as low as 130 $140^{\circ} \mathrm{C} / 50-60^{\circ} \mathrm{C}$, respectively (3): it consists of distributing the evaporation of water between several stages of drying, making it possible to reduce the drying kinetics and thus the drying temperatures as the drying time is increased. The first drying stage (chamber) leads to particle formation from the original droplets: their skin and morphology is formed here, but they are still carrying 5-10\% of residual moisture given the mild drying stress. The second drying stage (belt drying or crystalliser) finishes the water removal; in the case of high sugar content matrices, crystallization is obtained almost instantaneously given the high solids content, making it possible to remove the water of crystallization. Last, a fluidized bed drying stage can be used to cooling the powders and reaching the stable zone of dry glassy matrices, matching with appropriate water activity $\left(\mathrm{a}_{\mathrm{w}}\right.$ in the range $\left.0.05-0.1\right)$ and remaining below glass transition temperature.

\section{Conclusion}

The world market demand for functional microorganismscontaining foods and for food supplements is presently experiencing an exponential growth. This happens in a 

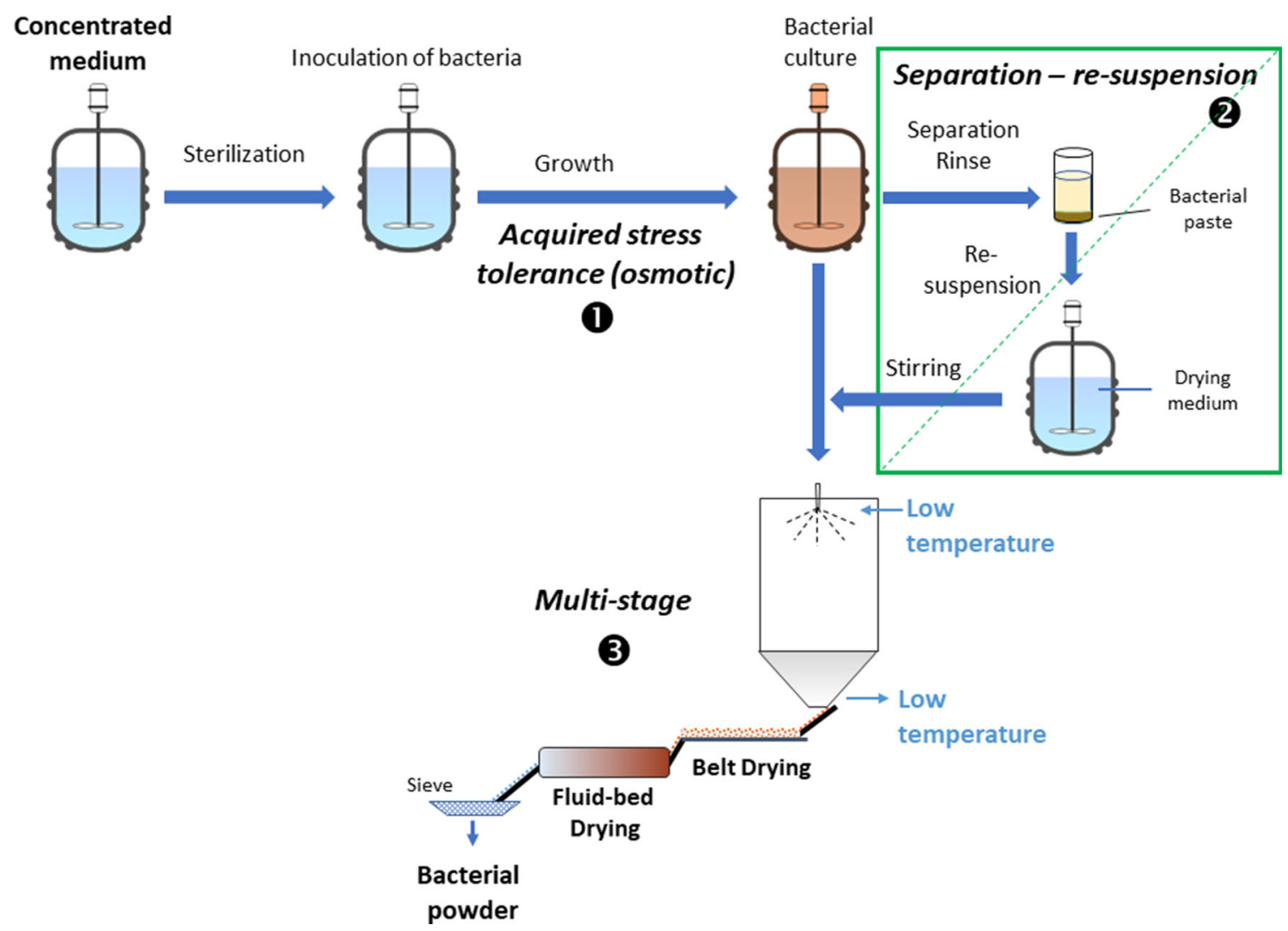

Fig. 2 Technological route for obtaining viable dry microorganisms. (1) Growth on concentrated medium makes it possible to enhance stress tolerance taking advantage of osmoadaptation. (2) Stress adapted bacteria can be separated, rinsed and re-suspended in a drying medium compatible with the market applications; the culture can either be directly

context of growing standard of living and growing preoccupation for the maintenance of health and comfort. Spray drying is very promising for sustainable and large-scale production of powders rich in probiotic and/or starter bacteria. However, while it is widely used in the dairy field, its use in the probiotic field is still restricted by the loss of bacterial viability. Improving the efficacy of beneficial bacteria spraydrying thus merits more efforts in research and development in order to better master bacteria physiological adaptation to such a process.

Concerning the dairy propionibacterium $P$. freudenreichii, a thorough investigation of bacterial adaptation, both during growth and after growth, recently opened new avenues for efficient drying of this probiotic. Growth in sublethal stressing conditions or post-culture treatments (thermal or hyperosmotic) leads to accumulation of compatible solutes including glycine betaine and trehalose. The ratio between these solutes is readily adjusted, depending on the medium composition (carbon, nitrogen). This, in turn, allows improvement of either freeze drying of spray drying of $P$. freudenreichii, in terms of survival, depending on the chosen stressing conditions. New possibilities to implement this immunomodulatory probiotic in functional products are opened. spray dried, which makes the process simpler and the contamination risk lower. (3) Multistage drying, that consists of coupling single stage spray drying with a belt drying and a fluid bed drying step, makes it possible further to lower inlet/outlet air temperature and maximize survival, while circumventing stickiness occurrence

Indeed, probiotic bacteria susceptible to modulate the gut microbiota, and the immune system, towards preserved homeostasis, offer great perspectives. The demand for immunomodulatory bacteria, able to mitigate inflammation and to modulate the maturation of the immune response at early stages of life will increase. This is linked with the growing incidence of ailments related to immune disorders, such as atopy and inflammation in developed countries. In this context, the production of whey, functionalized by the growth of immunomodulatory probiotics, opens great perspectives of development, as whey constitutes a major ingredient of infant formulas. Propionibacteria, whose presence as a result of breast-feeding, was recently shown to correlate with low incidence of necrotizing enterocolitis (Colliou et al. 2017), may play a key role in this context.

Acknowledgements The authors thank Xiao Dong Chen, Song Huang and Floriane Gaucher for their enthusiasm in probiotic bacteria drying. They acknowledge Emma Roilles and Kato Misation for helpful discussions and advices.

Author contribution RJ and GJ both gathered the necessary bibliography and wrote the manuscript. 
Funding RJ and GJ are funded by INRAE and by Institut Agro, French public research and teaching organisms.

\section{Declarations}

Ethics approval and consent to participate This article does not contain any studies with human participants or animals performed by any of the authors.

Conflict of interest GJ declares that he has no conflict of interest. RJ declares that he has no conflict of interest.

\section{References}

Al-Zoreky N, Ayres JW, Sandine WE (1991) Antimicrobial activity of Microgard $^{\mathrm{TM}}$ against food spoilage and pathogenic microorganisms1. J Dairy Sci 74:758-763. https://doi.org/10.3168/jds.S00220302(91)78222-2

Blaak EE, Canfora EE, Theis S, Frost G, Groen AK, Mithieux G, Nauta A, Scott K, Stahl B, van Harsselaar J, van Tol R, Vaughan EE, Verbeke K (2020) Short chain fatty acids in human gut and metabolic health. Benefic Microbes 11:411-455. https://doi.org/10.3920/ BM2020.0057

Bouglé D, Roland N, Lebeurrier F, Arhan P (1999) Effect of propionibacteria supplementation on fecal bifidobacteria and segmental colonic transit time in healthy human subjects. ScandJGastroenterol 34:144-148

Boyaval P, Deborde C, Corre C, Blanco C, Begue E (1999) Stress and osmoprotection in propionibacteria. Lait 79:59-69

Broeckx G, Vandenheuvel D, Claes IJJ, Lebeer S, Kiekens F (2016) Drying techniques of probiotic bacteria as an important step towards the development of novel pharmabiotics. Int J Pharm 505:303-318. https://doi.org/10.1016/j.ijpharm.2016.04.002

Cardoso FS, Castro RF, Borges N, Santos H (2007) Biochemical and genetic characterization of the pathways for trehalose metabolism in Propionibacterium freudenreichii, and their role in stress response. Microbiology 153:270-280

Colliou N, Ge Y, Sahay B, Gong M, Zadeh M, Owen JL, Neu J, Farmerie WG, Alonzo F, Liu K, Jones DP, Li S, Mohamadzadeh M (2017) Commensal Propionibacterium strain UF1 mitigates intestinal inflammation via Th17 cell regulation. J Clin Invest 127:3970-3986. https://doi.org/10.1172/JCI95376

Cousin FJ, Foligne B, Deutsch SM, Massart S, Parayre S, Le Loir Y, Boudry G, Jan G (2012a) Assessment of the probiotic potential of a dairy product fermented by Propionibacterium freudenreichii in piglets. JAgricFood Chem 60:7917-7927

Cousin FJ, Louesdon S, Maillard MB, Parayre S, Falentin H, Deutsch SM, Boudry G, Jan G (2012b) The first dairy product exclusively fermented by Propionibacterium freudenreichii: a new vector to study probiotic potentialities in vivo. Food Microbiol 32:135-146

Cousin FJ, Jouan-Lanhouet S, Theret N, Brenner C, Jouan E, Le MoigneMuller G, Manche-Boitrel MT, Jan G (2016) The probiotic Propionibacterium freudenreichii as a new adjuvant for TRAILbased therapy in colorectal cancer. Oncotarget

Deptula P, Kylli P, Chamlagain B, Holm L, Kostiainen R, Piironen V, Savijoki K, Varmanen P (2015) BluB/CobT2 fusion enzyme activity reveals mechanisms responsible for production of active form of vitamin B12 by Propionibacterium freudenreichii. Microb Cell Factories 14:186. https://doi.org/10.1186/s12934-015-0363-9

Deutsch S-M, Mariadassou M, Nicolas P, Parayre S, Le Guellec R, Chuat V, Peton V, Le Maréchal C, Burati J, Loux V, Briard-Bion V, Jardin J, Plé C, Foligné B, Jan G, Falentin H (2017) Identification of proteins involved in the anti-inflammatory properties of
Propionibacterium freudenreichii by means of a multi-strain study. Sci Rep 7:46409. https://doi.org/10.1038/srep46409

Dherbecourt J, Bourlieu C, Maillard MB, Ubert-Frogerais L, Richoux R, Thierry A (2010a) Time course and specificity of lipolysis in Swiss cheese. JAgricFood Chem 58:11732-11739

Dherbecourt J, Falentin H, Jardin J, Maillard MB, Bagliniere F, BarloyHubler F, Thierry A (2010b) Identification of a secreted lipolytic esterase in Propionibacterium freudenreichii, a ripening process bacterium involved in Emmental cheese lipolysis. ApplEnvironMicrobiol 76:1181-1188

FAO/WHO (2002) Guidelines for the evaluation of probiotics in food. Geneva, Switz: Food and Agriculture Organization of the United Nations and World Health Organization Working Group Report. p

Fitz A (1878) Ueber Spaltpilzgährungen. Ber Dtsch Chem Ges 11:18901899. https://doi.org/10.1002/cber.187801102180

FLR d C, Rabah H, Cordeiro BF, da Silva SH, Pessoa RM, SOA F, Cardoso VN, Gagnaire V, Deplanche M, Savassi B, Figueiroa A, Oliveira ER, Fonseca CC, MIA Q, Rodrigues NM, de Sandes SHC, Nunes ÁC, Lemos L, de Alves JL, AMC F, Ferreira Ê, Le Loir Y, Jan G, Azevedo V (2019) Probiotic Propionibacterium freudenreichii requires SlpB protein to mitigate mucositis induced by chemotherapy. Oncotarget 10:7198-7219. https://doi.org/10. 18632/oncotarget. 27319

Foligné B, Deutsch SM, Breton J, Cousin FJ, Dewulf J, Samson M, Pot B, Jan G (2010) Promising immunomodulatory effects of selected strains of dairy propionibacteria as evidenced in vitro and in vivo. ApplEnvironMicrobiol 76:8259-8264

Foligné B, Breton J, Mater D, Jan G (2013) Tracking the microbiome functionality: focus on Propionibacterium species. Gut 62:12271228

Fröhlich-Wyder MT, Bachmann HP (2004) Cheeses with propionic acid fermentation. In: Fox PF, McSweeney PLH, Cogan TM, Guinee TP (eds) Cheese: Chemistry, Physics and Microbiology. Academic Press, pp 141-XV

Fu N, Chen XD (2011) Towards a maximal cell survival in convective thermal drying processes. Food Res Int 44:1127-1149. https://doi. org/10.1016/j.foodres.2011.03.053

Furuichi K, Hojo K, Katakura Y, Ninomiya K, Shioya S (2006) Aerobic culture of Propionibacterium freudenreichii ET-3 can increase production ratio of 1,4-dihydroxy-2-naphthoic acid to menaquinone. JBiosciBioeng 101:464-470

Gaucher F, Bonnassie S, Rabah H, Leverrier P, Pottier S, Jardin J, BriardBion V, Marchand P, Jeantet R, Blanc P, Jan G (2019a) Benefits and drawbacks of osmotic adjustment in Propionibacterium freudenreichii. J Proteome 204:103400. https://doi.org/10.1016/j. jprot.2019.103400

Gaucher F, Gagnaire V, Rabah H, Maillard M-B, Bonnassie S, Pottier S, Marchand P, Jan G, Blanc P, Jeantet R (2019b) Taking advantage of bacterial adaptation in order to optimize industrial production of dry Propionibacterium freudenreichii. Microorganisms 7. https://doi. org/10.3390/microorganisms 7100477

Gaucher F, Kponouglo K, Rabah H, Bonnassie S, Ossemond J, Pottier S, Jardin J, Briard-Bion V, Marchand P, Blanc P, Jeantet R, Jan G (2019c) Propionibacterium freudenreichii CIRM-BIA 129 Osmoadaptation coupled to acid-adaptation increases its viability during freeze-drying. Front Microbiol 10:2324. https://doi.org/10. 3389/fmicb.2019.02324

Gaucher F, Rabah H, Kponouglo K, Bonnassie S, Pottier S, Dolivet A, Marchand P, Jeantet R, Blanc P, Jan G (2020) Intracellular osmoprotectant concentrations determine Propionibacterium freudenreichii survival during drying. Appl Microbiol Biotechnol 104:3145-3156. https://doi.org/10.1007/s00253-020-10425-1

Ge Y, Gong M, Colliou N, Zadeh M, Li J, Jones DP, Li S, Mohamadzadeh M (2019) Neonatal intestinal immune regulation by the commensal bacterium, P. UF1. Mucosal Immunol 12:434444. https://doi.org/10.1038/s41385-018-0125-1 
Hervé C, Fondrevez M, Cheron A, Barloy-Hubler F, Jan G (2007) Transcarboxylase mRNA: a marker which evidences $P$. freudenreichii survival and metabolic activity during its transit in the human gut. Int J Food Microbiol 113:303-314

Hojo K, Yoda N, Tsuchita H, Ohtsu T, Seki K, Taketomo N, Murayama T, Lino H (2002) Effect of ingested culture of Propionibacterium freudenreichii ET-3 on fecal microflora and stool frequency in healthy females. BiosciMicrofl 21:115-120

Huang S, Cauty C, Dolivet A, Le Loir Y, Chen XD, Schuck P, Jan G, Jeantet R (2016a) Double use of highly concentrated sweet whey to improve the biomass production and viability of spray-dried probiotic bacteria. J Funct Foods 23:453-463

Huang S, Rabah H, Jardin J, Briard-Bion V, Parayre S, Maillard M-B, Le Loir Y, Chen XD, Schuck P, Jeantet R, Jan G (2016b) Hyperconcentrated sweet whey, a new culture medium that enhances Propionibacterium freudenreichii stress tolerance. Appl Environ Microbiol 82:4641-4651. https://doi.org/10.1128/AEM. 00748-16

Huang S, Méjean S, Rabah H, Dolivet A, Le Loir Y, Chen XD, Jan G, Jeantet R, Schuck P (2017) Double use of concentrated sweet whey for growth and spray drying of probiotics: towards maximal viability in pilot scale spray dryer. J Food Eng 196:11-17

Hugenholtz J, Hunik J, Santos H, Smid E (2002) Nutraceutical production by propionibacteria. Lait 82:103-112

Iida K, Ohtaka K, Kajiwara M (2007) Mechanism of the ring contraction process in vitamin B12 biosynthesis by the anaerobe Propionibacterium shermanii under aerobic conditions. FEBS J 274:3475-3481. https://doi.org/10.1111/j.1742-4658.2007.05880.x

Isawa K, Hojo K, Yoda N, Kamiyama T, Makino S, Saito M, Sugano H, Mizoguchi C, Kurama S, Shibasaki M, Endo N, Sato Y (2002) Isolation and identification of a new bifidogenic growth stimulator produced by Propionibacterium freudenreichii ET-3. Biosci Biotechnol Biochem 66:679-681

Jan G, Rouault A, Maubois JL (2000) Acid stress susceptibility and acid adaptation of Propionibacterium freudenreichii subsp. shermanii. Lait 80:325-336

Jan G, Leverrier P, Pichereau V, Boyaval P (2001) Changes in protein synthesis and morphology during acid adaptation of Propionibacterium freudenreichii. ApplEnvironMicrobiol 67: 2029-2036

Jan G, Belzacq AS, Haouzi D, Rouault A, Metivier D, Kroemer G, Brenner C (2002a) Propionibacteria induce apoptosis of colorectal carcinoma cells via short-chain fatty acids acting on mitochondria. Cell Death Differ 9:179-188

Jan G, Leverrier P, Roland N (2002b) Survival and beneficial effects of propionibacteria in the human gut: in vivo and in vitro investigations. Lait 82:131-144

Lan A, Bruneau A, Philippe C, Rochet V, Rouault A, Hervé C, Roland N, Rabot S, Jan G (2007) Survival and metabolic activity of selected strains of Propionibacterium freudenreichii in the gastrointestinal tract of human microbiota-associated rats. Br J Nutr 97:714-724

Lan A, Bruneau A, Bensaada M, Philippe C, Bellaud P, Rabot S, Jan G (2008) Increased induction of apoptosis by Propionibacterium freudenreichii TL133 in colonic mucosal crypts of human microbiota-associated rats treated with 1,2-dimethylhydrazine. BrJ Nutr 100:1251-1259

Le Lay C, Coton E, Le Blay G, Chobert J-M, Haertlé T, Choiset Y, Van Long NN, Meslet-Cladière L, Mounier J (2016) Identification and quantification of antifungal compounds produced by lactic acid bacteria and propionibacteria. Int J Food Microbiol 239:79-85. https:// doi.org/10.1016/j.ijfoodmicro.2016.06.020

Le Marechal C, Peton V, Ple C, Vroland C, Jardin J, Briard-Bion V, Durant G, Chuat V, Loux V, Foligne B, Deutsch SM, Falentin H, Jan G (2015) Surface proteins of Propionibacterium freudenreichii are involved in its anti-inflammatory properties. JProteomics 113C: $447-461$
Leverrier P, Dimova D, Pichereau V, Auffray Y, Boyaval P, Jan G (2003) Susceptibility and adaptive response to bile salts in Propionibacterium freudenreichii: physiological and proteomic analysis. ApplEnvironMicrobiol 69:3809-3818

Leverrier P, Vissers JP, Rouault A, Boyaval P, Jan G (2004) Mass spectrometry proteomic analysis of stress adaptation reveals both common and distinct response pathways in Propionibacterium freudenreichii. ArchMicrobiol 181:215-230

Lind H, Jonsson H, Schnurer J (2005) Antifungal effect of dairy propionibacteria - contribution of organic acids. Int J Food Microbiol 98:157-165

Lind H, Sjögren J, Gohil S, Kenne L, Schnürer J, Broberg A (2007) Antifungal compounds from cultures of dairy propionibacteria type strains. FEMS Microbiol Lett 271:310-315. https://doi.org/10.1111/ j.1574-6968.2007.00730.x

McIntosh FM, Shingfield KJ, Devillard E, Russell WR, Wallace RJ (2009) Mechanism of conjugated linoleic acid and vaccenic acid formation in human faecal suspensions and pure cultures of intestinal bacteria. Microbiology 155:285-294

Mitsuyama K, Masuda J, Yamasaki H, Kuwaki K, Kitazaki S, Koga H, Uchida M, Sata M (2007) Treatment of ulcerative colitis with milk whey culture with Propionibacterium freudenreichii. JIntestMicrobiol 21:143-147

Mizote A, Yamada M, Yoshizane C, Arai N, Maruta K, Arai S, Endo S, Ogawa R, Mitsuzumi H, Ariyasu T, Fukuda S (2016) Daily intake of trehalose is effective in the prevention of lifestyle-related diseases in individuals with risk factors for metabolic syndrome. J Nutr Sci Vitaminol (Tokyo) 62:380-387. https://doi.org/10.3177/jnsv.62. 380

Pasteur L, Faulkner F (1879) Studies on fermentation: the diseases of beer, their causes, and the means of preventing them. Kraus Reprint

Penedo LA, Nunes JC, Gama MAS, Leite PEC, Quirico-Santos TF, Torres AG (2013) Intake of butter naturally enriched with cis9, trans 11 conjugated linoleic acid reduces systemic inflammatory mediators in healthy young adults. J Nutr Biochem 24:2144-2151. https://doi.org/10.1016/j.jnutbio.2013.08.006

Piveteau P (1999) Metabolism of lactate and sugars by dairy propionibacteria: a review. Lait 79:23-41

Plé C, Richoux R, Jardin J, Nurdin M, Briard-Bion V, Parayre S, Ferreira S, Pot B, Bouguen G, Deutsch S-M, Falentin H, Foligné B, Jan G (2015) Single-strain starter experimental cheese reveals antiinflammatory effect of Propionibacterium freudenreichii CIRM BIA 129 in TNBS-colitis model. J Funct Foods 18:575-585. https://doi.org/10.1016/j.jff.2015.08.015

Plé C, Breton J, Richoux R, Nurdin M, Deutsch S-M, Falentin H, Hervé C, Chuat V, Lemée R, Maguin E, Jan G, Van de Guchte M, Foligné B (2016) Combining selected immunomodulatory Propionibacterium freudenreichii and Lactobacillus delbrueckii strains: reverse engineering development of an anti-inflammatory cheese. Mol Nutr Food Res 60:935-948. https://doi.org/10.1002/ mnfr.201500580

Rabah H, Rosa do Carmo FL, Jan G (2017) Dairy Propionibacteria: versatile probiotics. Microorganisms 5. https://doi.org/10.3390/ microorganisms5020024

Rabah H, Ferret-Bernard S, Huang S, Le Normand L, Cousin FJ, Gaucher F, Jeantet R, Boudry G, Jan G (2018) The cheese matrix modulates the immunomodulatory properties of Propionibacterium freudenreichii CIRM-BIA 129 in healthy piglets. Front Microbiol 9:2584. https://doi.org/10.3389/fmicb.2018.02584

Rabah H, FLR d C, de Carvalho RDO, Cordeiro BF, da Silva SH, Oliveira ER, Lemos L, Cara DC, AMC F, Garric G, Harel-Oger M, Le Loir Y, Azevedo V, Bouguen G, Jan G (2020) Beneficial propionibacteria within a probiotic emmental cheese: impact on dextran sodium sulphate-induced colitis in mice. Microorganisms 8:380. https://doi.org/10.3390/microorganisms 8030380 
Schuck P, Dolivet A, Méjean S, Hervé C, Jeantet R (2013) Spray drying of dairy bacteria: new opportunities to improve the viability of bacteria powders. IntDairy Journal 31:12-17

Seki K, Nakao H, Umino H, Isshiki H, Yoda N, Tachihara R, Ohuchi T, Saruta H, Suzuki K, Mitsuoka T (2004) Effects of fermented milk whey containing novel bifidogenic growth stimulator produced by Propionibacterium on fecal bacteria, putrefactive metabolite, defecation frequency and fecal properties in senile volunteers needed serious nursing-care taking enteral nutrition by tube feeding. JIntestMicrobiol 18:107-115

Sniffen JC, McFarland LV, Evans CT, Goldstein EJC (2018) Choosing an appropriate probiotic product for your patient: an evidence-based practical guide. PLoS One 13:e0209205. https://doi.org/10.1371/ journal.pone.0209205

Suomalainen T, Sigvart-Mattila P, Matto J, Tynkkynen S (2008) In vitro and in vivo gastrointestinal survival, antibiotic susceptibility and genetic identification of Propionibacterium freudenreichii ssp shermanii JS. IntDairy Journal 18:271-278

Thierry A, Maillard MB, Yvon M (2002) Conversion of L-leucine to isovaleric acid by Propionibacterium freudenreichii TL 34 and ITGP23. ApplEnvironMicrobiol 68:608-615

Thierry A, Maillard MB, Hervé C, Richoux R, Lortal S (2004) Varied volatile compounds are produced by Propionibacterium freudenreichii in Emmental cheese. Food Chem 87:439-446
Thierry A, Falentin H, Deutsch SM, Jan G (2011) Propionibacterium spp. In: Fuquay JW FP and MP (ed), 1st edn. Academic Press, San Diego, pp 403-411

Turgay M, Bachmann HP, Irmler S, von Ah U, Fröhlich-Wyder MT, Falentin H, Deutsch SM, Jan G, Thierry A (2020) Propionibacterium spp. and Acidipropionibacterium spp. $\}_{3}$. In: Encyclopedia of Dairy Science. Elsevier

Viladomiu M, Hontecillas R, Bassaganya-Riera J (2016) Modulation of inflammation and immunity by dietary conjugated linoleic acid. Eur J Pharmacol 785:87-95. https://doi.org/10.1016/j.ejphar.2015.03. 095

von Freudenreich E, Orla-Jensen O (1906) Uber die in Emmentalerkäse stattfindene Propionsäure-gärung. ZentralblBakteriol 17:529-546

Yee AL, Maillard MB, Roland N, Chuat V, Leclerc A, Pogacic T, Valence F, Thierry A (2014) Great interspecies and intraspecies diversity of dairy propionibacteria in the production of cheese aroma compounds. IntJFood Microbiol 191:60-68

Yuan G, Chen X, Li D (2015) Modulation of peroxisome proliferatoractivated receptor gamma (PPAR $\gamma$ ) by conjugated fatty acid in obesity and inflammatory bowel disease. J Agric Food Chem 63: 1883-1895. https://doi.org/10.1021/jf505050c

Publisher's note Springer Nature remains neutral with regard to jurisdictional claims in published maps and institutional affiliations. 